\title{
A home-based training program improves Taiwanese family caregivers' quality of life and decreases their risk for depression: a randomized controlled trial
}

\author{
Li-Min Kuo $^{1, \dagger}$, Huei-Ling Huang ${ }^{2, \dagger}$, Hsiu-Li Huang ${ }^{3}$, Jersey Liang ${ }^{4}$, Yi-Chen Chiu ${ }^{5}$, Sien-Tsong Chen ${ }^{6}$, \\ Yam-Ting Kwok ${ }^{7}$, Wen-Chuin $\mathrm{Hsu}^{8}$ and Yea-Ing L. Shyu ${ }^{5}$ \\ ${ }^{1}$ School of Nursing, Hsin Sheng College of Medical Care and Management, Taoyuan, Taiwan \\ ${ }^{2}$ Department of Gerontological Care and Management, Chang Gung University of Science and Technology, Taoyuan, Taiwan \\ ${ }^{3}$ School of Nursing, Chang Gung University, Taoyuan, Taiwan \\ ${ }^{4}$ School of Public Health and Institute of Gerontology, University of Michigan, Ann Arbor, MI, USA \\ ${ }^{5}$ School of Nursing and Healthy Aging Research Center, Chang Gung University, Taoyuan, Taiwan \\ ${ }^{6}$ Department of Neurology, Chang Gung Memorial Hospital, Taoyuan, Taiwan \\ ${ }^{7}$ Division of Neurology, Far Eastern Memorial Hospital, Taipie, Taiwan \\ ${ }^{8}$ School of Medicine, Chang Gung University, Taoyuan, Taiwan \\ Correspondence to: Yea-Ing L. Shyu, E-mail: yeaing@mail.cgu.edu.tw
}

${ }^{\dagger}$ The first two authors contributed equally to this article.

Objectives: Little is known about the longitudinal effects of training programs on family caregivers' health-related quality of life (HRQoL) and depressive symptoms over time. Therefore, the purpose of this study was to examine the effects of a home-based caregiver training program on HRQoL and depressive symptoms for family caregivers of older persons with dementia.

Methods: Outcomes (caregivers' HRQoL and depressive symptoms) were assessed before the training program (baseline), and at 2 weeks, 3 months, and 6 months afterwards. HRQoL was measured using the Medical Outcomes Study 36-item Short Form Survey, Taiwan version. Depressive symptoms were measured using the Chinese version Center for Epidemiologic Studies Depression Scale.

Results: Family caregivers who received the individualized home-based training program had better health outcomes in bodily pain $(b=12.37, p<0.013)$, role disability due to emotional problems $(b=17.74, p<0.013)$, vitality $(b=12.40, p<0.001)$, better mental summary score $(b=5.14, p<0.003)$, and decreased risk for depression (odds ratio $=0.15$, confidence interval $=0.04-0.65, p<0.013$ ) than those in the control group during the 6 months following the training program.

Conclusion: Our home-based caregiver training program improved caregivers' HRQoL, especially role limitations due to emotional problems, and decreased their risk for depression. Copyright (C) 2012 John Wiley \& Sons, Ltd.

Key words: dementia; behavioral symptoms; health-related quality of life; depression; home-based caregiver training program History: Received 27 September 2011; Accepted 8 June 2012; Published online 9 July 2012 in Wiley Online Library

(wileyonlinelibrary.com)

DOI: 10.1002 /gps.3853

\section{Introduction}

Individuals with dementia commonly exhibit behavioral and psychological symptoms. Indeed, $90 \%$ of these patients in Western developed countries experience at least one behavioral and psychological symptom during the course of the disease (Prado-Jean et al., 2010). Many patients with dementia (75\%) have neuropsychiatric symptoms such as delusion, anxiety, and depression (Lyketsos et al., 2002). Furthermore, $9 \%-51.5 \%$ of Western patients with dementia exhibit agitation, persecution, wandering, and aggression (Lyketsos et al., 2002; Savva et al., 2009; Prado-Jean et al., 2010). Among Taiwanese patients with dementia, 30\%-79.3\% displayed 
psychiatric symptoms, 35\%-76\% had anxiety, and $31 \%-57 \%$ had aggressive behaviors (Shyu et al., 1994; Fuh et al., 2005). Such problem behaviors increase the burden on family caregivers, with adverse effects on their mental health and quality of life (Davis, 1997), as well as physical health (von Känel et al., 2010).

Health outcomes for family caregivers have been shown to improve after participation in communitybased caregiver training programs on handling dementia patients' behavioral problems. For example, caregivers' depressive symptoms improved after a group education intervention (Gallagher-Thompson et al., 2001) and home visits with or without telephone consultation and support (Bormann et al., 2009; Elliott et al., 2010). Family caregivers' quality of life and psychosocial health also improved after participating in education and support groups (Fung and Chien, 2002; Chien and Lee, 2008; Gavrilova et al., 2009; Elliott et al., 2010; Chien and Lee, 2011). Furthermore, a metaanalysis found that caregivers' psychological well-being, depression, and social outcomes improved after support group interventions, including mutual support, psycho-education, and education (Chien et al., 2011). However, most of these studies were conducted in Western developed countries, with only three Asian studies, all conducted in Hong Kong (Fung and Chien, 2002; Chien and Lee, 2008; Chien and Lee, 2011). In addition, few of these studies described longitudinal changes in intervention effects.

In Taiwan, a pilot study showed that a home-based caregiver training program for family caregivers of older patients with dementia significantly decreased patients' behavioral problems, but not their physically aggressive behaviors and improved caregivers' self-efficacy in a 3 -month period (Huang et al., 2003). However, the study sample was recruited from a single hospital, the intervention was delivered by a single research nurse, the study did not explore the effects of the training program on caregivers' health-related quality of life (HRQoL) and depressive symptoms; and outcomes were only explored over 3 months (Huang et al., 2003). Therefore, we modified that training program by adding a component of service utilization consultation, by including multiple research nurses to deliver the intervention, and by recruiting caregivers from a wider variety of sources (two hospitals and a community care-management center). The intervention provided caregivers with skills and strategies to lower environmental stresses for the person with dementia in their care. The home-based caregiver training program emphasized the partnership between healthcare providers and family caregivers, helped caregivers identify the stresses that caused behavioral problems, and developed appropriate environmental support and case-management services. We hypothesized that after receiving this training program, caregivers' HRQoL would improve and their risk for depression would decrease.

\section{Methods}

\section{Design}

A single-blinded randomized clinical trial was used to explore the effects of the caregiver training program on family caregivers' HRQoL, depression, preparation for dementia care, appropriate strategies for managing behavioral problems, and satisfaction with management of behavioral problems. After random assignment, caregivers in the experimental group received the community-based caregiver training program with telephone consultations, and participants in the control group received only written educational materials and social telephone follow-ups. Data were collected on HRQoL, depression, preparation for dementia care, appropriate strategies for managing behavioral problems, and satisfaction with management of behavioral problems at 2 weeks, 3 months, and 6 months after the experimental group had completed the training program.

\section{Participants}

Patients were included if they met the following criteria: (i) diagnosed with dementia by a psychiatrist or neurologist; (ii) 65 years or older; (iii) living in a home in northern Taiwan; and (iv) scored $\geq 50$ on the Cohen-Mansfield Agitation Inventory, indicating having behavioral problems (Huang et al., 2003).

Of 374 patients with dementia screened in the neurological clinics of two hospitals and a community care-management center in northern Taiwan, 251 met the inclusion criteria. These 251 people with dementia and their caregivers were invited to participate in this study; $129(51.39 \%)$ of them agreed to participate and were randomly assigned to the experimental $(n=63)$ or control group $(n=66)$. During the study period, $21(16.28 \%)$ participating patients were lost to follow-up because of further refusal $(n=17)$, institutionalization $(n=1)$, change of family caregiver $(n=2)$, and death $(n=1)$. At the end of 6 -month follow-up, 108 people with dementia and their family caregivers (55 and 53 caregiver-care receiver dyads in the experimental and control groups, respectively) remained in the study (Figure 1). 


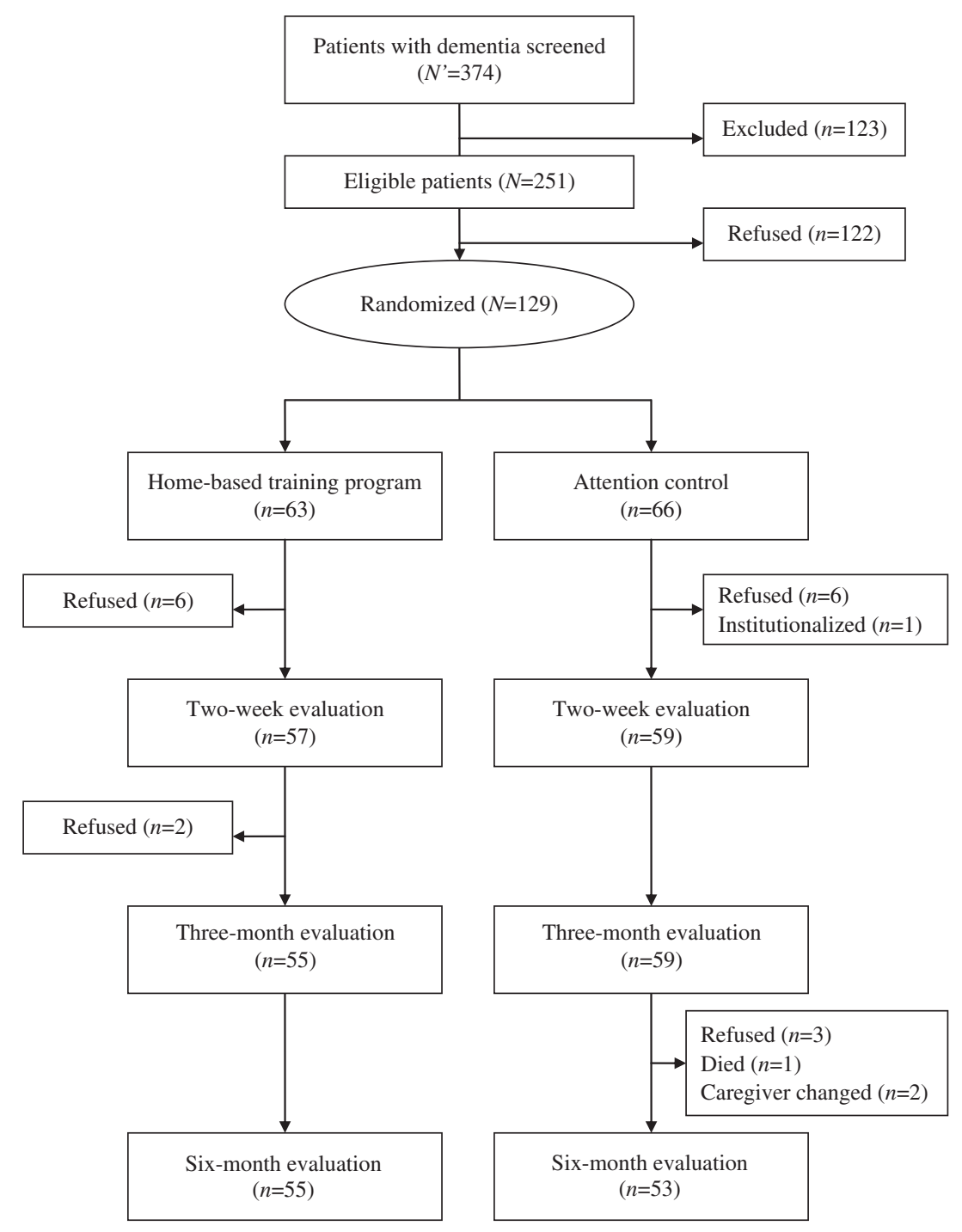

Figure 1 Study flow chart.

\section{Caregiver training program}

Theoretical framework. The theoretical basis for our family caregiver training program integrated the Progressively Lowered Stress Threshold (PLST) model (Hall and Buckwalter, 1987), the concept of partnership with family caregivers (Harvath et al., 1994), and the Antecedent Event-Behavior-Consequence (ABC) theory (Skinner, 1953). The PLST model proposes that patients with dementia show anxious or agitated behaviors due to the demands of environmental and internal stimuli and to increasing dysfunction resulting from progressive cerebral pathology and associated cognitive decline. The training program emphasized helping caregivers to identify the causative stressors of patients' specific behavioral problems and provided suggestions to modify the environment and daily schedule to decrease stress.

Skinner's ABC theory, which focuses on antecedents and consequences of a specific behavior (Skinner, 1953), was used to help family caregivers manage the identified specific behavioral problems of patients with dementia. Family caregivers were taught to explore and change what happens directly before or directly after the problem behavior, to alter or at least decrease its frequency.

The concept of partnership with family caregivers emphasizes the importance of combining nurses' and family caregivers' knowledge about managing patients' behavioral problems because the caregiver has important knowledge about the patient (Harvath et al., 1994). On the basis of this notion, our training program combined 
the nurses' and caregivers' knowledge not only to identify behavioral problems and their causes but also to plan for the patient's individual care, including assessment and referral to community services needed by the family caregiver.

Intervention protocol. Using this theoretical framework, we developed a two-session, in-home training program, with each session 1 week apart (Table 1). At the initial visit, which lasted 2 to $3 \mathrm{~h}$, a research nurse visited the home to establish a partnership with the family caregiver and to conduct a structured assessment of the dementia patient's condition. Then the research nurse worked with the family caregiver to identify the targeted behavioral problems and to explore the causative environmental stimuli, antecedents, and consequences of the targeted behavioral problems. Strategies were offered to provide positive reinforcement to enhance the desired behavior. A tentative plan was made with the caregiver to minimize these stimuli and decrease the targeted behavioral problems by modifying the daily schedule and environment.

In the second session, lasting 2 to $3 \mathrm{~h}$ as well, the nurse visited the family caregiver to further assess family resources, strengths and weaknesses, provide information regarding dementia care, confirm the patients' behavioral problems, and finalize the plan for handling specific behavioral problems. The plan included the identified behavioral problems, specific environmental stimuli, and agreed-upon handling strategies based on the first assessment. Specific suggestions were reemphasized and adjusted if needed to modify the environment to lower causative environmental stimuli. On the basis of each caregiver's needs, the nurse gave information and made referrals to community services.

At 1 week after the second visit and once a month thereafter, the research nurse made follow-up phone calls, each lasting $20-30 \mathrm{~min}$, to family caregivers to find out the conditions of the targeted behavioral problems and to evaluate progress in managing those problems. If referrals to community services were made in prior visits, the nurse also evaluated utilization of those services.

\section{Outcome variables}

HRQoL. Family caregivers' HRQoL was measured by the Taiwan version of the Medical Outcomes SF-36 (Lu et al., 2003; Tseng et al., 2003). The SF-36 is a widely used profile measure of generic HRQoL, thus allowing further comparison among patients across different countries and/or with different diseases (Weinberger et al., 1991; Lyons et al., 1994). The SF-36 has 36 items representing eight generic health concepts: physical functioning $(\mathrm{PF})$, role disability due to physical health problems (RP); bodily pain (BP); vitality (energy/fatigue) (VT); general health perceptions $(\mathrm{GH})$; role disability due to emotional problems (RE); social functioning (SF); and general mental health (MH). Mental component summary (MCS) and physical component summary (PCS) scores were calculated using norm-based $(50,10)$ scoring methods (Ware et al., 1994) with Taiwan-specific SF-36 algorithms (McHorney, 1996). Scores for each scale range from 0 to 100 , with higher scores representing better health outcomes. The SF-36 has been reported to have good validity and reliability among older populations in the United States (Weinberger et al., 1991; Lyons et al., 1994; McHorney, 1996). The SF-36 was translated into Chinese (Taiwan version) and demonstrated to have good reliability and validity (Tseng et al., 2003). In this study, Cronbach's alphas for the eight scales ranged from 0.75 to 0.97 .

Depressive symptoms. Risk for depression was measured by the Chinese version of the Center for Epidemiologic Studies Depression Scale (CES-D; Radloff, 1977), a widely used instrument for measuring depressive symptomatology in the general population. The CES-D contains 20 items representing symptoms whose frequency in the previous week is rated on a scale from

Table 1 The training program

\begin{tabular}{|c|c|c|}
\hline Schedule & Experimental group & Control group \\
\hline Initial visit & $\begin{array}{l}\text { 1. Assess the dementia patient, her/his family caregiver, environment, } \\
\text { and needs for community resources. } \\
2 \text {. Assess specific behavioral problems and develop a tentative } \\
\text { behavioral management plan. }\end{array}$ & $\begin{array}{l}\text { Provide written educational } \\
\text { materials. }\end{array}$ \\
\hline $\begin{array}{l}\text { Second visit (1 week after } \\
\text { initial visit) }\end{array}$ & $\begin{array}{l}\text { 1. Provide care plan and positive reinforcement strategies for } \\
\text { specific behavioral problems. } \\
\text { 2. Provide information and referrals to community services. }\end{array}$ & - \\
\hline $\begin{array}{l}\text { Phone call ( } 1 \text { week after } \\
\text { second visit) }\end{array}$ & $\begin{array}{l}\text { Follow targeted behavioral problems and utilization of } \\
\text { community services. }\end{array}$ & Telephone social contact \\
\hline $\begin{array}{l}\text { Phone calls (monthly from } \\
\text { first to sixth month) }\end{array}$ & Telephone consultations & Telephone social contact \\
\hline
\end{tabular}


0 (less than 1 day), to 1 (1-2 days), 2 (3-4 days), and 3 (5-7 days). The CES-D has been translated and demonstrated to have good psychometric properties including reliability, validity, sensitivity, and specificity in Chinese and Taiwanese samples (Fu et al., 2003). CES-D scores $\geq 15$ indicate risk for depression (Chien and Cheng, 1985). In this study, Cronbach's alpha for the CES-D was 0.93 .

Preparation for dementia care, appropriate strategies for managing behavioral problems, and satisfaction with management of behavioral problems. "Preparation for dementia care" was assessed using the Caregiver Preparedness Scale (Huang, 2001); "appropriate strategies for managing behavioral problems," and "satisfaction with management of behavioral problems" were assessed using the Caregivers Competence of Behavioral Problem Management Scale (Huang and Shyu, 2003). Preparation for dementia care was scored on a 5-point Likert scale from 1 (unprepared) to 5 (well prepared). Appropriate strategies for managing behavioral problems and satisfaction with management of behavioral problems were scored for frequency on a 5-point Likert scale from 1 (never) to 5 (always).

\section{Procedure}

This study was approved by the Human Subjects Protection Committee (97-1850B) of Chang Gung Memorial Hospital in Taiwan. Research assistants approached eligible family caregivers at the outpatient clinics of two hospitals in Taipei and cases referred by the local care-management center to explain the study and participants' right to withdraw. Those who agreed to participate signed the written consent and were randomly assigned to the experimental or control group.

Both groups were assessed at baseline (before the intervention) for HRQoL and depressive symptoms. The experimental group received the caregiver training program, and the control group received printed sheets with general information on dementia such as the causes, courses, and symptoms of dementia, but no specific information on handling behavioral problems. At 2 weeks, 3 months, and 6 months after the experimental group completed the two-session training program, both groups received a follow-up assessment for HRQoL and depressive symptoms. To minimize attrition, caregivers in both groups received monthly follow-up phone calls from the first to the sixth month.

\section{Statistical analysis}

Analyses were carried out under an intention-to-treat principle. The results using this principle were similar to those using on-protocol subjects. Changes in outcome variables were analyzed using hierarchical linear models (Raudenbush and Bryk, 2002). Because the variables were normally distributed, general hierarchical generalized linear models were applied for eight HRQoL scales, PCS, MCS, preparation for dementia care, appropriate strategies for managing behavioral problems, and satisfaction with management of behavioral problems. CES-D scores $<15$ were categorized as 0 (without risk for depression), and CES-D scores $\geq 15$ were categorized as 1 (at risk for depression). Thus, hierarchical generalized linear models with dichotomous dependent variables were appropriate. We chose to specify the control group as the reference category. Doing so allowed us to estimate the odds of risk for depression in the experimental group relative to the odds for the control group over time. For all analyses, we centered time at 6 months after the caregiving program was completed to minimize the possibility of multicollinearity when evaluating nonlinear changes with time (Raudenbush and Bryk, 2002). Finally, attrition was accounted for by using a dummy variable for subjects who dropped out in this study. In addition, we controlled for attrition for each outcome variable by treating its attrition as a covariate. Considering the issue of multiple comparisons in this study, we adjusted the significance levels for $p$-values from $0.05,0.01$, and 0.001 to $0.013,0.003$, and 0.001 , respectively, by the Bonferroni method (Bland and Altman, 1995; Savitz and Olshan, 1995). Outcome variables were measured at baseline, 2 weeks, 3 months, and 6 months following the intervention, but are plotted in Figure 2 according to regression coefficients that account for all parameters in the multi-level analysis, not the raw scores.

\section{Results}

\section{Baseline participant characteristics}

Of the 129 patients with dementia who participated in this study, $54.3 \%$ were female, with an average age of 80.26 years $(\mathrm{SD}=6.95)$. For clinical characteristics, $52.7 \%$ had Alzheimer's disease, 35.7\% had vascular dementia, and the time since diagnosis with dementia was 46.09 months $(\mathrm{SD}=37.00)$. In terms of dementia severity as measured by the Clinical Dementia Rating Scale (Morris, 1993; Lin and Liu, 2003), 36.4\% patients had mild dementia, $34.1 \%$ had moderate dementia, and $29.5 \%$ had severe dementia. For care receivers' cognitive status as measured by the Chinese MiniMental State Examination (Yip et al., 1997), their average score was $10.95(\mathrm{SD}=7.07)$, representing 
A

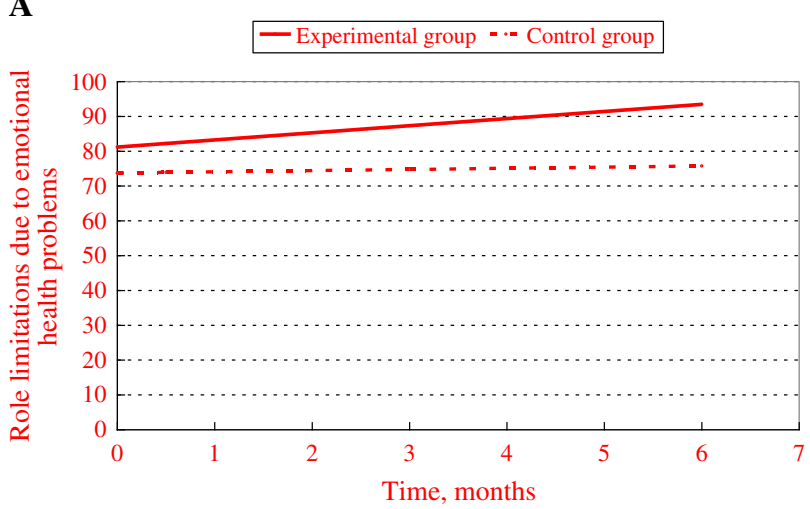

B

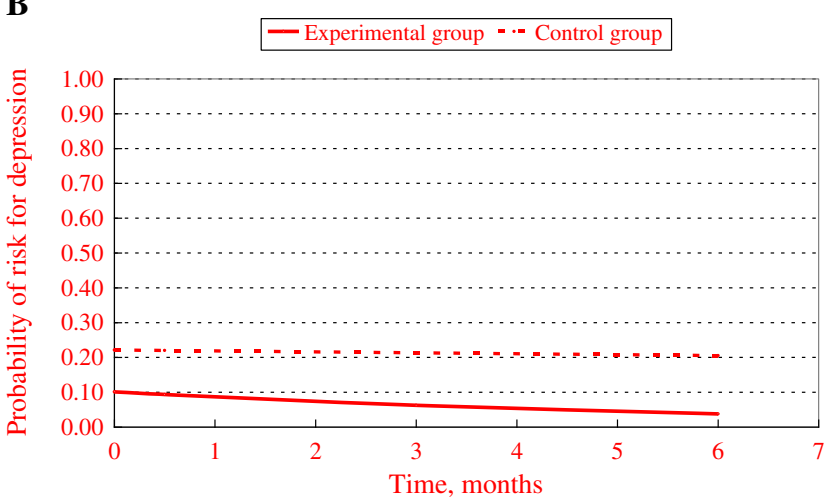

Figure 2 Time course of improvement in (A) caregivers' role limitations due to emotional health problems (score range $=0-100$; higher scores indicate better HRQoL) and (B) probability of risk for depression (CES-D scores $\geq 15$ indicate risk for depression) by group.

moderate dementia. These characteristics of patients with dementia did not differ significantly by group.

Among the family caregivers, $75.2 \%$ were female, with a mean age of 55.47 years $(\mathrm{SD}=13.69)$. The largest proportion of caregivers had completed high school $(35.7 \%)$, and they were almost equally distributed among spouses $(28.7 \%)$, daughters $(27.9 \%)$, and daughters-inlaw $(27.1 \%)$. The rest were sons $(16.3 \%)$. The caregivers spent on average $13.45 \mathrm{~h}(\mathrm{SD}=8.39)$ per day caring for dementia patients. They had been family caregivers for an average of 42.63 months $(S D=36.16)$. Only $34.9 \%$ hired a home helper. Caregivers in the experimental and control groups did not differ significantly in any characteristics (Table 2).

\section{Outcome comparisons}

Family caregivers who received the individualized home-based training program had better health outcomes in bodily pain ( $b=12.37, p<0.013)$, role disability due to emotional problems $(b=17.74, p<0.013)$, vitality $(b=12.40, p<0.001)$, better mental summary score $(b=5.14, p<0.003)$, and decreased risk for depression (odds ratio $=0.15$, confidence interval $=$ $0.04-0.65, p<0.013)$ than those in the control group during the 6 months following the training program (Table 3). At the same time, these caregivers were better preparation for dementia care $(b=0.41, p<0.013)$, had more appropriate strategies for managing behavioral problems $(b=0.94, p<0.001)$, and were more satisfied with their management of behavioral problems $(b=0.79, p<0.001)$ than control group caregivers over the 6 months after the training program.

In other words, at 6 months following the intervention, caregivers who received the training program had 12.37 points more in $\mathrm{BP}, 17.74$ points more in RE, 12.40 points more in VT, and 5.14 points more in MCS (on possible scores ranging from 0 to 100) than those in the control group. At the same time, family caregivers who received the individualized homebased training program were less likely to be at risk for depression ( $\mathrm{OR}=0.15, \mathrm{CI}=0.04-0.65, p<0.013$ ), indicating $85 \%$ lower likelihood of risk for depression than those in the control group during the 6 months following the training program. Family caregivers in the experimental group had 0.41 points more in preparation for dementia care, 0.94 points more in implementing appropriate strategies for managing behavioral problems, and 0.79 points more in their satisfaction with management of behavioral problems (on possible scores ranging from 1 to 5 ). These results suggest that the effects of the intervention lasted over 6 months after the training program ended. In particular, the intervention effects on vitality and risk for depression increased during the follow-up period. The control group had relatively stable role disability because of emotional problems and risk of depression during the 6-month follow-up, whereas RE scores continuously increased and risk of depression decreased in the experimental group during the same period (Figure 2).

\section{Discussion}

This study found that a home-based caregiver training program, which was developed from the PLST model and $\mathrm{ABC}$ theory and used a partnership with family caregivers of patients with dementia, significantly improved caregivers' HRQoL and decreased their likelihood of risk for depression, with effects lasting at least 6 months. The intervention helped caregivers identify the stresses that caused behavioral problems in patients with dementia and helped caregivers develop appropriate environmental support and case 
Table 2 Comparison of caregivers' demographic and caregiving characteristics by group

\begin{tabular}{|c|c|c|c|}
\hline Characteristic & Experimental group $(n=63)$ & Control group $(n=66)$ & $\boldsymbol{p}^{*}$ \\
\hline Age (years), mean $\pm S D$ & $54.73 \pm 15.10$ & $56.17 \pm 12.28$ & 0.55 \\
\hline Gender, $n(\%)$ & & & 0.51 \\
\hline Male & $14(22.2)$ & $18(27.3)$ & \\
\hline Female & $49(77.8)$ & $48(72.7)$ & \\
\hline Educational background, $n(\%)$ & & & 0.07 \\
\hline Illiterate & $3(4.8)$ & $6(9.1)$ & \\
\hline Primary school & $11(17.5)$ & $23(34.8)$ & \\
\hline High school & $23(36.5)$ & $23(34.8)$ & \\
\hline College & $12(19.0)$ & $6(9.1)$ & \\
\hline University or above & $14(22.2)$ & $8(12.1)$ & \\
\hline Relationship to care receiver & & & 1.00 \\
\hline Spouse & $18(28.6)$ & $19(28.8)$ & \\
\hline Son & $10(15.9)$ & $11(16.7)$ & \\
\hline Daughter & $18(28.6)$ & $18(27.3)$ & \\
\hline Daughter-in-law & $17(27.0)$ & $18(27.3)$ & \\
\hline Time caring for patients per day (hours), mean $\pm S D$ & $12.07 \pm 8.17$ & $14.74 \pm 8.45$ & 0.72 \\
\hline Duration of caregiving (months), mean $\pm S D$ & $38.48 \pm 29.00$ & $46.53 \pm 41.64$ & 0.21 \\
\hline Hired helper & & & 0.99 \\
\hline Yes & $22(34.9)$ & $23(34.8)$ & \\
\hline No & $41(65.1)$ & $43(65.2)$ & \\
\hline
\end{tabular}

$S D=$ standard deviation;

${ }^{*} p$-values are based on analysis of variance or Pearson chi-square test.

management services. This study expanded our prior study (Huang et al., 2003) with a more comprehensive intervention and examined the effect of the intervention on more long-term outcomes.

The PLST model has been shown to be effective in interventions to decrease depression among caregivers of patients with dementia (Buckwalter et al., 1999), decrease caregiving impact (Stolley et al., 2002), and improve caregiver's immune function (Garand et al., 2002). On the other hand, the ABC model was shown to be useful in anger treatment (Fuller et al., 2010). However, little is known regarding its effects on health outcomes of family caregivers of patients with dementia (Volicer and Hurley, 2003).

This randomized, controlled study showed that integrating the PLST and ABC approaches was effective in developing a caregiver training program that improved health outcomes of family caregivers of patients with dementia in Taiwan. The clinical significance of these improvements in HRQoL outcomes can be assessed by the minimally important difference (MID), which indicates the smallest difference in score for the domain of interest that is perceived as beneficial. A MID of 5 is suggested for the SF-36 (Walters, 2004; Bjorner et al., 2007). In this study, the difference between the experimental and control groups in BP, $\mathrm{RE}$, and VT were close to or greater than 10; and in MCS, it was greater than 5, indicating a clinically significant effect of the intervention on caregivers' HRQoL. Thus, the intervention program was more clinically efficient in improving caregivers' bodily pain, vitality, general health perception, and social function than for other health-related outcomes.

This study also found that the intervention program decreased caregivers' risk for depression by $85 \%$ compared with that of control caregivers of patients with dementia. This result is consistent with reports that home visits with or without telephone consultation and support effectively decreased depressive symptoms of family caregivers of dementia patients (Bormann et al., 2009; Elliott et al., 2010). In this study, the intervention effect on decreasing risk for depression lasted for at least 6 months after ending the home-based training program.

Despite its contributions, this study had some limitations. First, the study used a single-blinded design, that is, only patients with dementia and their family caregivers were blinded to which intervention they received. However, the impact of this limitation may have been minimized by caregivers' self-reporting the outcome variables. Second, we did not collect information on caregivers' life events that might have influenced the results. On the other hand, this issue might have been addressed by randomly assigning caregivers to groups, which assumes that the characteristics of the experimental and control groups are equivalent (D'Agostino and D'Agostino, 2007). Third, the control group had less contact with a health professional than the intervention group, which might have exaggerated the intervention effects. Finally, the study 


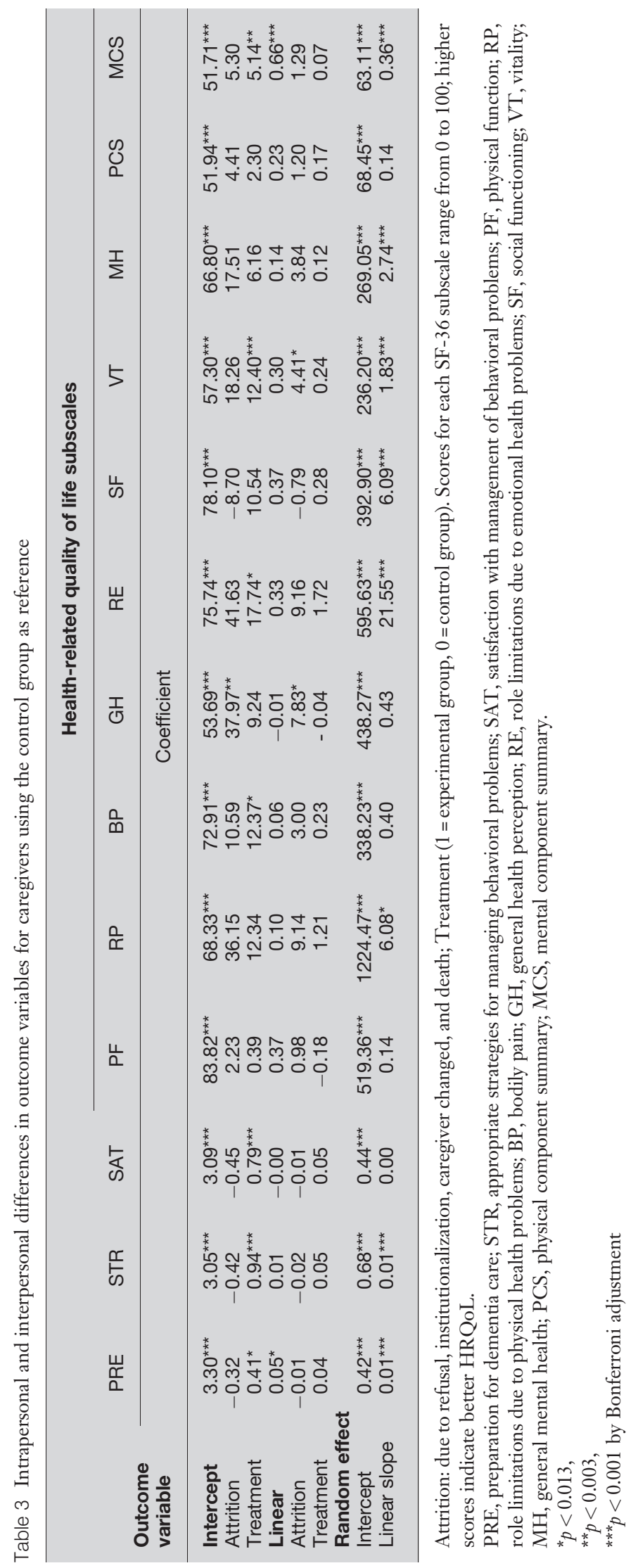


sample was small. However, this limitation was mitigated by the study's statistical power, which was sufficient to detect meaningful treatment effects (Leon, 2008). Caregivers' RE had a power of 0.99 and their BP, VT, and MCS had a power of 0.93 at 6 months following the intervention (Diggle et al., 1994; Liu and Wu, 2005; Brown and Prescott, 2006).

In conclusion, this training program in its current form, which combines PLST and ABC approaches, effectively improved health outcomes and decreased risk of depression in a Taiwanese sample of family caregivers of patients with dementia. Similar programs may be applicable to other countries with Chinese populations, and the results of this study can provide a reference for healthcare providers who deal with Chinese/Taiwanese immigrants.

\section{Conflict of interest}

None declared.

\section{Key points}

- Our randomized clinical trial study shows that the two-session in-home caregiver training program, which integrated the PLST model and $\mathrm{ABC}$ theory, is helpful for improving caregivers' health-related quality of life, especially role limitations due to emotional problems, and it decreases their risk for depression.

- Similar programs may be applicable to other countries with Chinese populations, and the results of this study can provide a reference for health care providers who deal with Chinese/ Taiwanese immigrants.

\section{Acknowledgement}

This work was supported by grant NSC 97-2420-H182-002-MY3 from the National Science Councils of Taiwan, ROC.

\section{References}

Bjorner JB, Wallenstein GV, Martin MC, et al. 2007. Interpreting score differences in the SF-36 Vitality scale: using clinical conditions and functional outcomes to define the minimally important difference. Curr Med Res Opin 23(4): 731-739. DOI: $10.1185 / 030079907 X 178757$

Bland JM, Altman DG. 1995. Multiple significance tests: the Bonferroni method. BMJ 310(6973): 170.
Bormann J, Warren KA, Regalbuton L, et al. 2009. A spiritually based caregiver intervention with telephone delivery for family caregivers of veterans with dementia. Fam Community Health 32(4): 345-353.

Brown H, Prescott R. 2006. Applied Mixed Models in Medicine. 2nd Ed. Wiley: Chichester, West Sussex, England.

Buckwalter KC, Gerdner L, Kohout F, et al. 1999. A nursing intervention to decrease depression in family caregivers of persons with dementia. Arch Psychiat Nurs 13(2): 80-88. DOI: 10.1016/S0883-9417(99)80024-7

Chien CP, Cheng TA. 1985. Depression in Taiwan: epidemiological survey utilizing CES-D. Seishin Shinkeigaku Zasshi 87(5): 335-338.

Chien WT, Lee YM. 2008. A disease management program for families of persons in Hong Kong with dementia. Psychiatr Serv 59(4): 433-436. DOI: 10.1176/appi. ps.59.4.433

Chien WT, Lee IY. 2011. Randomized controlled trial of a dementia care programme for families of home-resided older people with dementia. J Adv Nurs 67(4): 774-787. DOI: $10.1111 /$ j.1365-2648.2010.05537.x

Chien LY, Chu H, Guo JL, et al. 2011. Caregiver support groups in patients with dementia: a meta-analysis. Int J Geriatr Psychiatry 26(10): 1089-1098. DOI:10.1002/gps.2660

Davis LL. 1997. Family conflicts around dementia home-care. Fam Syst Health 15(1): 85-98. DOI: $10.1037 / \mathrm{h} 0089810$

D'Agostino RB, D'Agostino RS. 2007. Estimating treatment effects using observational data. JAMA 297(3): 314-316.

Diggle PG, Liang KY, Zeger SL. 1994. Analysis of Longitudinal Data. Oxford University Press: New York, NY.

Elliott AF, Burgio LD, DeCoster J. 2010. Enhancing caregiver health: findings from the resources for enhancing Alzheimer's caregiver health intervention. $J \mathrm{Am}$ Geriatr Soc 58(1): 30-37. DOI: 10.1111/j.1532-5415.2009.02631.x

Fu CC, Lee YM, Chen JD. 2003. Association between depressive symptoms and twelve-year mortality among elderly in a rural community in Taiwan. J Formos Med Assoc 102(4): 234-239.

Fuh JL, Wang SJ, Cummings JL. 2005. Neuropsychiatric profiles in patients with Alzheimer's disease and vascular dementia. J Neurol Neurosurg Psychiatry 76(10): 1337-1341. DOI: 10.1136/jnnp.2004.056408

Fuller JR, Digiuseppe R, O'Leary S, Fountain T, Lang C. 2010. An open trial of a comprehensive anger treatment program on an outpatient sample. Behav Cogn Psychoth 38(4): 485-90. DOI: 10.1017/S1352465810000019

Fung WY, Chien WT. 2002. The effectiveness of a mutual support group for family caregivers of a relative with dementia. Arch Psychiatr Nurs 16(3): 134-144. DOI: 10.1053/apnu.2002.32951

Gallagher-Thompson D, Arean P, Rivera P, Thompson LW. 2001. A psychoeducational intervention to reduce distress in Hispanic family caregivers: Result of a pilot study. Clin Gerontol 23(1/2): 17-32. DOI: 10.1300/J018v23n01_03

Garand L, Buckwalter KC, Lubaroff DM, et al. 2002. A pilot study of immune and mood outcomes of a community-based intervention for dementia caregivers: The PLST Intervention. Arch Psychiatr Nurs 16(4): 156-167. DOI: 10.1053/ apnu.2002.34392

Gavrilova SI, Ferri CP, Mikhaylova N, et al. 2009. Helping carers to care-The 10/66 Dementia research group's randomized control trail of a caregiver intervention in Russia. Int J Geriatr Psychiatry 24(4): 347-354. DOI: 10.1002/gps.2126

Hall GR, Buckwalter KC. 1987. Progressively lowered stress threshold: a conceptual model for care of adult with Alzheimer's disease. Arch Psychiatr Nurs 1(6): 399-406.

Harvath TA, Archbold PG, Stewart BJ, et al. 1994. Establishing partnerships with family caregivers. Local and cosmopolitan knowledge. J Gerontol Nurs 20(2): 29-35.

Huang HL. 2001. The Effectiveness of a Community-based Caregiver Training Program for Improving the Caregiver's Competence and Decreasing the Behavioral Problems of Elders with Dementia [dissertation]. Chang Gung University: Taoyuan, Taiwan. (Chinese)

Huang HL, Shyu YI. 2003. The effectiveness of a community-based caregiver training program for improving the caregiver's competence of elders with dementia. $J$ of Long-Term Care 7: 121-134. (Chinese)

Huang HL, Shyu YI, Chen MC, Chen ST, Lin LC. 2003. A pilot study on a home-based caregiver training program for improving caregiver self-efficacy and decreasing the behavioral problems of elders with dementia in Taiwan. Int J Geriatr Psychiatry 18(4): 337-45. DOI: $10.1002 / \mathrm{gps} .835$

Leon AC. 2008. Implications of clinical trial design on sample size requirements. Schizophrenia Bull 34(4): 664-69. DOI: 10.1093/schbul/sbn035

Lin KN, Liu HC. 2003. Clinical dementia rating (CDR): Chinese version. Acta Neurol Taiwan 12(3): 154-165.

Liu $\mathrm{H}, \mathrm{Wu}$ T. 2005. Sample size calculation and time-averaged difference. JMASM 4(2): 434-45.

Lu JR, Tseng HM, Tsai YJ. 2003. Assessment of health-related quality of life in Taiwan (I): development and psychometric testing of SF-36 Taiwan version. Taiwan J Public Health 22(6): 501-511.

Lyketsos CG, Lopez O, Jones B, et al. 2002. Prevalence of neuropsychiatric symptoms in dementia and mild cognitive impairment. JAMA 288(12): 1475-1483. DOI: 10.1001/jama.288.12.1475 
Lyons RA, Perry HM, Littlepage BN. 1994. Evidence for the validity of the Short Form 36 Questionnaire (SF-36) in an elderly population. Age Ageing 23(3): 182-184. DOI: 10.1093/ageing/23.3.182

McHorney CA. 1996. Measuring and monitoring general health status in elderly persons: practical and methodological issues in using the SF-36 Health Survey. Gerontologist 36(5): 571-583. DOI: 10.1093/geront/36.5.571

Morris JC. 1993. The Clinical Dementia Rating (CDR): current version and scoring rules. Neurology 43(11): 2412-2414.

Prado-Jean A, Couratier P, Druet-Cabanac M, et al. 2010. Specific psychological and behavioral symptoms of depression in patients with dementia. Int J Geriatr Psychiatry 25(10): 1065-1072. DOI: 10.1002/gps.2468

Radloff LS. 1977. The CES-D scale: a self-report depression scale for research in the general population. Appl Psychol Meas 1(3): 385-401. DOI: 10.1177/ 014662167700100306

Raudenbush SW, Bryk AS. 2002. Hierarchical Linear Models: Applications and Data Analysis Methods. 2nd Ed. Sage: Newbury Park, CA.

Savitz DA, Olshan AF. 1995. Multiple comparisons and related issues in the interpretation of epidemiologic data. Am J Epidemiol 142(9): 904-908.

Savva GM, Matthews FE, Davidson JE, Davidson JE, et al. 2009. Prevalence, correlates and course of behavioural and psychological symptoms of dementia in the population. Br J Psychiatry 194(3): 212-219. doi: 10.1192/bjp.bp.108.049619

Shyu YI, Yip PK, Lee JY, Chen, R. 1994. An investigation of behavior problems of elderly persons with dementia in two communities of Taipei. Acta Neurol Sinica 3(1): 1-7.
Skinner BF. 1953. Science and Human Behavior. Free Press: New York.

Stolley JM, Reed D, Buckwalter KC. 2002. Caregiving appraisal and interventions based on the progressively lowered stress threshold model. Am J Alzheimers Dis Other Demen 17(2): 110-120. DOI: 10.1177/153331750201700211

Tseng HM, Lu JR, Tsai YJ. 2003. Assessment of health-related quality of life (II): norming and validation of SF-36 Taiwan version. Taiwan J Public Health 22(6): 512-518.

Volicer L, Hurley AC. 2003. Management of behavioral symptoms in progressive degenerative dementias. J Gerontol A Biol Sci Med Sci 58(9): M837-M845. DOI: 10.1093/gerona/58.9.M837

von Känel R, Ancoli-Israel S, Dimsdale JE, et al. 2010. Sleep and biomarkers of atherosclerosis in elderly Alzheimer caregivers and controls. Gerontology 56(1): 41-50. DOI: 10.1159/000264654

Walters SJ. 2004. Sample size and power estimation for studies with health related quality of life outcomes: a comparison of four methods using the SF-36. Health Qual Life Outcomes 2: 26. DOI: 10.1186/1477-7525-2-26

Ware JE, Kosinski MK, Keller SD. 1994. SF-36 Physical and Mental Health Summary Scales: A User's Manual. The Health Institute, New England Medical Center: Boston.

Weinberger M, Samsa GP, Hanlon JT, et al. 1991. An evaluation of a brief health status measure in elderly veterans. I Am Geriatr Soc 39(7): 691-694.

Yip PK, Shyu YI, Liu SI. 1997. The multidisciplinary project of dementia study in northern Taiwan (DSNT) background and methodology. Acta Neurol Taiwan 6(3): 210-216. 